\title{
Numerical Control AC Servo System Based on PMSM
}

\author{
Mengxiong Zeng ${ }^{1}$, a , Jinfeng Zhao ${ }^{1, b}$ \\ ${ }^{1}$ College of Mechanical and Power Engineering, China Three Gorges University, Yichang, 443002, \\ China \\ aemail: zmxiong88@hotmail.com, bemail: jinfeng_zhao@foxmail.com
}

Keywords: Servo system; CNC; vector control; tuning

\begin{abstract}
The paper study object is the CNC servo system, and it presented three closed-loops control strategy based on PMSM. Through dynamic mathematical model of current loop, rotational speed loop and position loop were established and simplified. The closed-loop regulator was designed. The system was corrected by the controller. The simulation, tuning and calculation of the closed-loop were carried in MATLAB/SIMULINK. The results indicated that the system design was correct, and the desired effect of control was achieved.
\end{abstract}

\section{Introduction}

AC Control Systems are widely used in CNC field. However, its mathematical mode of high order multivariable; strong coupling and nonlinear characteristics make the control more complex. Engineering design often used vector transform method of the linear decoupling control based on Permanent Magnet Synchronous Motor ( PMSM ) CNC servo system. Then using regulator correction, parameters setting and optimization is to improve the dynamic performance of servo control system [1-2].

\section{CNC Servo System Control Structure}

The CNC System in industrial production is a kind of three-loop control structure AC servo system based on PMSM [3]. The system is composed with the current loop, the speed loop and the position loop from the inside to the outside. The current loop uses servo motor armature current as feedback quantity; it makes the torque of the motor adjust to set value, and it could improve the system's rapid and suppress the inner interference of current loop. The speed loop uses motor speed as feedback quantity, through controlling motor output speed to resist load disturbance and speed fluctuation, provide system with rapid accurate location and tracking. The position loop uses the control object linear displacement or angular displacement output as feedback quantity. The role of the position loop is to ensure the static accuracy and dynamic tracking performance of the system. The system outer-loop performance depends on the inner-loop optimization. The optimization of each loop performance is the basis of the high-performance servo system. Generally, in engineer designing, we start from the inner ring of regulator. Firstly, the inner loop of the regulator is designed. Secondly, the outer loop of the regulator is designed, in which the inner part of the whole as a controlling object. Then regulators of all the control loops will be designed gradually.

\section{Current Loop and Parameter Setting}

The current loop includes digital PI regulator, the time constant, the SVPWM inverter of amplification factor, the current detecting device with the low-pass filter and the feedback coefficients. The current loop block diagram is as Fig. 1. 


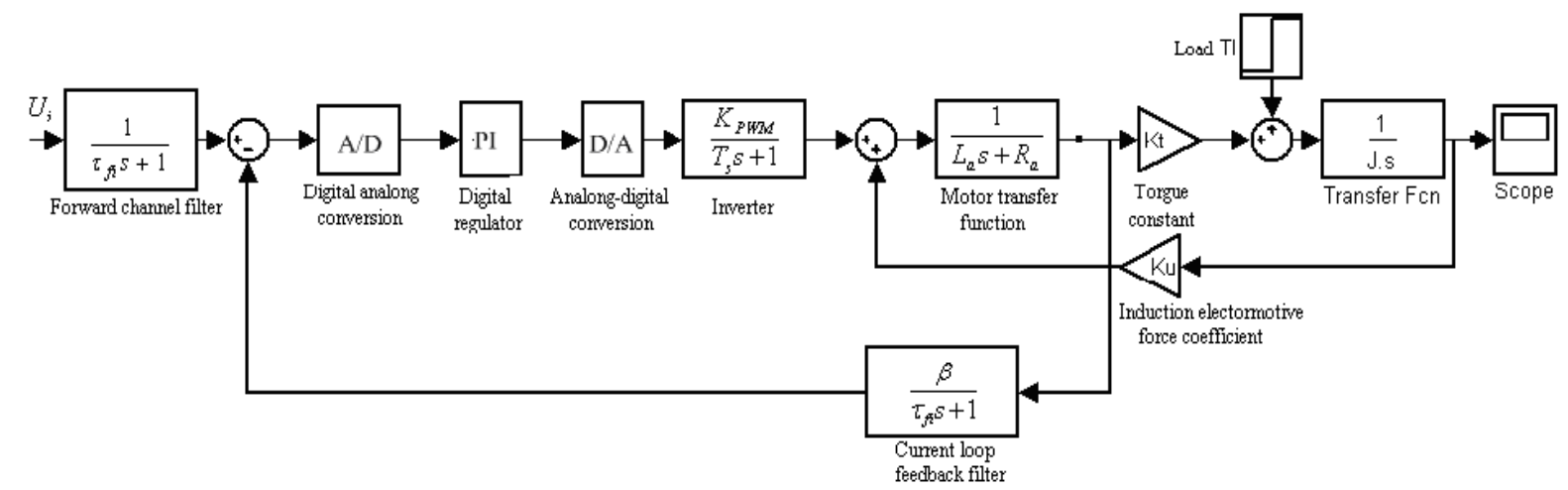

Figure 1 Current loop block diagram

To balance the effect of current feedback delay, a forward channel filter is added, of which is the same as the current feedback filter .It could make the set signal and the feedback signal have the same delay time. Because the electromagnetic time constant is much smaller than the mechanical time constant, we could consider that the feedback EMF is unchanged within the current transient process, neglect feedback EMF when design the current loop and then change the input signal to . As both the SVPWM inverter and the filter times constant are small, its countdown is in the high frequency band of logarithmic frequency characteristic, approximate treatment can't bring significantly influence on the dynamic performance of the system [4]. Based on the above reasons to simplify current loop, simplified current regulatory control object was consisted of two inertia links, the transfer function was as Eq. (1).

$$
G(s)=\frac{K_{1}}{\tau_{\Sigma i} s+1} \cdot \frac{1 / R_{a}}{\tau_{a} s+1}
$$

Where, $\tau_{\Sigma i}=\tau_{f i}+\tau_{0}, K_{1}=\beta K_{P W M}, \tau_{a}=L_{a} / R_{a}$. Servo control system expected that current loop was supposed to have no static error at steady state and keep the current do not exceed the allowable value in the dynamic process [5]. Based on these requirements, the current loop should be corrected as a typical I system. Its transfer function was as Eq. (2).

$$
G_{i}(z)=K_{P}+K_{I} \frac{T_{s}}{z-1}
$$

According to the simulation system, it could analysis the parameters tuning of time domain equation. Because of $\tau_{a}<\tau_{\Sigma i}$, in order to keep that the controller zero offset large time constants pole of control object, we chose $\tau_{a}=K_{P} / K_{I}$. So the current open-loop transfer function was as Eq. (3).

$$
G_{I}(s)=\frac{K_{i}}{s\left(\tau_{\Sigma i} s+1\right)}
$$

According to the "best" second-order system, we took $K_{i}=\frac{K_{P} \beta K_{P W M}}{R_{a} \tau_{a}}, K_{P}=\frac{R_{a} \tau_{a}}{2 \beta K_{P W M} \tau_{\Sigma i}}$, $K_{I}=R_{a} / 2 \beta \tau_{\Sigma i}$.

\section{Speed Loop and Parameter Setting}

Designing speed loop, the designed current loop was equivalent to a link of the speed control process and the controlled object. Because the current loop equivalents transfer function was a second order oscillation link as Eq. (4).

$$
\frac{I_{a}(s)}{U_{i}^{*}(s)}=\frac{1 / \beta}{2 \tau_{\Sigma i}^{2} s^{2}+2 \tau_{\Sigma i} s+1}
$$

The inherent frequency was $\omega_{n}=1 /\left(\sqrt{2} \tau_{\Sigma i}\right)$. The order of current loop transfer function was 
reduced as $\frac{I_{a}(s)}{U_{i}^{*}(s)}=\frac{1 / \beta}{2 \tau_{\Sigma i} s+1}$. The double inertial link is equivalent to a single inertia link with the small time constant $2 \tau_{\Sigma i}$; the current following performance is accelerated. The speed loop diagram of the dynamic structure is shown in Figure 2.

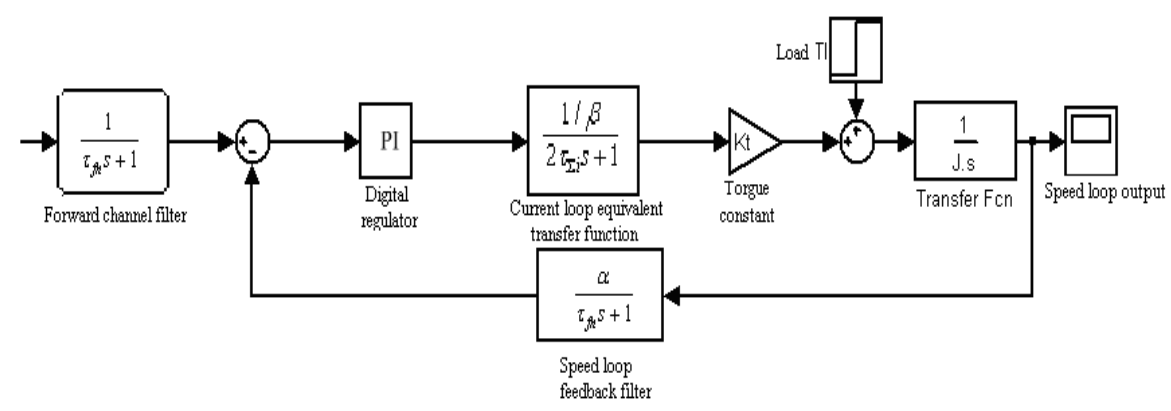

Figure 2 Speed loop dynamic block diagram

To ensure the speed control precision and response speed, the low-pass filter is added to the speed loop and the balance filter is added after a signal has been given in order the speed detection device feedback coefficient was $\alpha=1$. The feedback filter and forward channel filter equivalent moved to the inner loop. The given signal equivalent transform was $U_{n}^{*} / \alpha$. The small time constant $\tau_{\Sigma n}$ instead of $\tau_{f n}$ added $2 \tau_{\Sigma i}$, that was $\tau_{\Sigma n}=\tau_{f n}+2 \tau_{\Sigma i}$.

As a typical II type system, the speed loop should be designed to be a stable state without steady-state error and have a dynamic performance with a good disturbance restraint performance. The corresponding digital PI controller was as Eq. (5).

$$
G_{n}(z)=K_{p n}+K_{I n} \frac{T_{n}}{z-1}
$$

Where, $K_{p n}$ was speed regulator proportional coefficient, $K_{I n}$ was speed regulator differential coefficient, $T_{n}$ was speed loop sampling period.

In the simulation system the speed loop open-loop transfer function was as Eq. (6).

$$
G(s)=\frac{K_{n}\left(\frac{K_{p n}}{K_{I n}} s+1\right)}{s^{2}\left(\tau_{\Sigma n} s+1\right)}
$$

For the typical II type system, the parameters of the system were determined according to the minimum closed-loop amplitude frequency characteristics. According to the relationship among intermediate frequency bandwidth $h$, dynamic characteristic indicators and anti-jamming performance index, we took the best choice $h=5$.

$$
\begin{aligned}
& K_{p n} / K_{I n}=h \tau_{\Sigma n}=5 \tau_{\Sigma n} \\
& K_{n}=\frac{h+1}{2 h^{2} \tau_{\Sigma n}^{2}}
\end{aligned}
$$

Take Eq. (7) and Eq. (8) into Eq. (6) to get Eq. (9) and Eq. (10).

$$
\begin{aligned}
& K_{p n}=\frac{J \beta(h+1)}{2 h \tau_{\Sigma n} K_{t} \alpha} \\
& K_{I n}=\frac{K_{p n}}{5 \tau_{\Sigma n}}
\end{aligned}
$$

Torque constant $K_{t}$ was the proportion of rated torque and rated current $K_{t}=T_{N} / I_{N}$, so in the 
difference equations $u(n)=u(n-1)+\Delta u(n)$ of speed loop incremental digital PI controller was as Eq. (11).

$$
\begin{aligned}
\Delta u(n) & =K_{P_{n}}[e(n)-e(n-1)]+K_{I n} T_{n} e(n) \\
& =\frac{3 J \beta}{5 \tau_{\Sigma n} K_{t} \alpha}[e(n)-e(n-1)]+\frac{3 J \beta T_{n}}{25 \tau_{\Sigma n}^{2} K_{t} \alpha} e(n)
\end{aligned}
$$

\section{Position Loop and Parameter Setting}

The position loop of the servo system achieved precise positioning and fast tracking. In the position loop of CNC servo system, when the speed regulator uses the PI adjuster and the cut-off frequency of position loop is far less than the reciprocal of each speed loop time constant, the speed closed-loop transfer function is approximately equivalent to a first-order process. This treatment can better reflect the speed loop characteristic and simplify the design of position loop. $K_{p}$ was the proportional coefficient of position loop regulator; $K_{N}$ was the gain of speed loop; $K_{p 3}$ was proportional coefficient of the position detection loop. The position regulator was used as a typical type system. When using speed input as CNC following system given input signal, the steady-state error was as Eq. (12).

$$
\varepsilon=\frac{1}{K}=\frac{1}{K_{P} K_{N}}
$$

In the type, $K$ was to gain of position loop. The tracking error of servo system input signal is inversely proportional to the gain $K_{p}$ of position loop regulator. As $K_{p}$ is bigger, the position tracking error is smaller, but as $K_{p}$ is increasing, the stability of the servo system will be influenced. So setting $K_{p}$ should make all consideration for various requirements.

\section{Case and Analysis}

For a control object of PMSM, its parameters: rated power was $1.1 \mathrm{~kW}$, rated voltage was $220 \mathrm{~V}$, rated speed was $1000 \mathrm{r} / \mathrm{min}$, stator resistance was $2.875 \Omega$, inductance was $8.5 \mathrm{mH}$, rotational inertia was $0.0008 \mathrm{~kg} . \mathrm{m} 2$,PWM wave load frequency was $2.5 \mathrm{kHz}$,current loop filter time was $40 \mu \mathrm{s}$, speed loop filter time was $2 \mathrm{~ms}$. According to sampling period determining the principle, sampling period of current loop was $0.0005 \mathrm{~s}$; speed loop sampling period was 0.001 s.In the Vector Controlled PMSM System, both current loop regulator and speed loop regulator had adopted incremental digit PI regulator, parameters of current loop regulator:

$$
\begin{aligned}
& K_{P}=L_{a} / 2 \beta \tau_{\Sigma i}=9.66 \\
& K_{I}=K_{P} / \tau_{a}=R_{a} / 2 \beta \tau_{\Sigma i}=3267.05
\end{aligned}
$$

Speed loop parameters:

$$
\begin{aligned}
& K_{p n}=\frac{J \beta(h+1)}{2 h \tau_{\Sigma n} K_{t} \alpha}=\frac{3 J \beta}{5 \tau_{\Sigma n} K_{t} \alpha}=0.143 \\
& K_{I n}=\frac{K_{p n}}{5 \tau_{\Sigma n}}=\frac{3 J \beta}{25 \tau_{\Sigma n}{ }^{2} K_{t} \alpha}=10
\end{aligned}
$$

According to sampling period determining the principle, current loop sampling period was $0.0005 \mathrm{~s}$; speed loop sampling period was $0.001 \mathrm{~s}$. The simulation model could be established in MATLAB/SIMULINK. Under the different conditions with no-load starting, stable operation with load, suddenly loading, we could simulate and the obtained experimental data were in Table 1. 
Table 1 the Simulation Results under Different Condition

\begin{tabular}{|c|c|c|c|c|}
\hline & $\begin{array}{c}\text { Rise Time } \\
(\mathrm{ms})\end{array}$ & $\begin{array}{c}\text { Peak Time } \\
(\mathrm{ms})\end{array}$ & $\begin{array}{c}\text { Adjusting Time } \\
(\mathrm{ms})\end{array}$ & $\begin{array}{c}\text { Maximum Overshoot } \\
(\mathrm{MP})\end{array}$ \\
\hline Non-load starting & 14 & 17 & 36 & 8.7 \\
\hline Load starting (5N.m) & 25 & 29 & 48 & 3.1 \\
\hline $\begin{array}{c}\text { Non-load Disturbance } \\
\text { (5N.m) }\end{array}$ & 14 & 17 & 36 & 8.7 \\
\hline
\end{tabular}

The results had shown, under non-load starting, current waveform of torque and stator had certain wave in start-up phase, but the speed ripple was small after the stable operation. When operation with load is stable, the speed ripple was very small and stator current had good sine characteristics. During the stable operation, sudden load was given in appropriate moment, the speed ripple was small and stator current ran with good sine characteristics. Those illustrated the system had good fast-response and anti-interference ability of the system, the rightness of the whole system design was verified and the desired results were attained.

\section{References}

[1] Ruan Yi, Chen Boshi. Electric Drive Automatic Control System -- Motion Control System (Fourth Edition) [M]. Beijing: Mechanical Industry Press. 2010

[2] Ruan Yi, Chen Weijun. Motion Control System[M]. Beijing: Tsinghua University Press, 2007

[3] Zeng Mengxiong, Fang Chunjiao. Designing and Tuning Current Loop on AC Servo System Base on PMSM[J]. Modular Machine Tool \& Automatic Manufacturing Technique, 2011(7):44-49

[4] Wang Yang. Simulation of PMSM with Vector Control Based on Simulation[J].Modular Machine Tool \& Automatic Manufacturing Technique, 2011(2):78-5

[5] Zeng Mengxiong, Zhao Jinfeng, Ouyang Wen. PID Parameter Fuzzy Self-Tuning of the Motion Control System[J]. Process Equipment, Mechatronics Engineering and Material Science, 2014(6):187-191 